\section{The hydroxyapatite orbital implant: post-operative pain}

\begin{abstract}
Purpose To compare and quantify the degree of post-operative pain experienced by patients undergoing primary and secondary hydroxyapatite orbital implantation. Methods A prospective survey design was employed to monitor patients' pain preoperatively and up to 7 days post-operatively. Fifty-five consecutive patients undergoing primary and secondary hydroxyapatite orbital implantation were recruited over a period of 1 year. Standardised anaesthetic, operative and post-operative protocols were followed.

During the patients' in-patient stay nurses completed a data proforma that required them to record patients' pain using an 11-item numerical rating scale. A self-completion proforma was given to patients to score their pain at home until the first post-operative outpatient appointment.

Results Patients undergoing primary implant surgery tend to experience more post-operative pain than those undergoing secondary implant surgery. A small proportion of patients experience severe post-operative pain. Conclusion Post-operative pain experienced by patients undergoing primary and secondary hydroxyapatite implantation has been underestimated. This study has provided the necessary data to enable such patients to
\end{abstract}

H. Waterman

B. Leatherbarrow

R. Slater

Manchester Royal Eye

Hospital

Manchester, UK

H. Waterman

C. Waterman

School of Nursing

University of Manchester

Manchester, UK

\section{Hillier}

Medical Computation University of Manchester Manchester, UK

Brian Leatherbarrow Manchester Royal Eye

Hospital

Oxford Road

Manchester M13 9PH, UK

Fax: $+44(0) 1612726618$ Key words Hydroxyapatite orbital implant, Postoperative pain

The hydroxyapatite orbital implant is an integrated buried implant made from marine coral that is inserted following enucleation or evisceration. ${ }^{1}$ Its structure resembles that of human cancellous bone, being very porous. Good motility of the artificial eye is achieved by the attachment of extraocular muscles to the implant. This permits fibrovascular ingrowth from the muscles. If necessary the motility of the artificial eye can be improved through direct coupling to the hydroxyapatite implant by means of a motility peg, which is inserted into a
HEATHER WATERMAN, BRIAN

LEATHERBARROW, ROGER SLATER,

CHRISTINE WATERMAN, VAL HILLIER hole drilled in the implant at a second procedure performed some months later. ${ }^{2}$ Studies have shown that the hydroxyapatite orbital implant has a low rate of infection, extrusion and migration. ${ }^{2-5}$ Hydroxyapatite spheres used both as primary and secondary orbital implants have been shown to provide good cosmesis and excellent motility of the artificial eye with a low rate of complications. ${ }^{3}$

It has been our experience that some patients encounter severe post-operative pain following hydroxyapatite implantation. We investigated this post-operative pain experience in order to provide adequate pre-operative patient counselling and to plan a rational discharge policy, against a background of increasing pressure to discharge patients early following surgery.

\section{Patients and methods}

A prospective descriptive surgery design was employed to address the study aim. Following approval from the local ethics committee, all consecutive patients admitted to Manchester Royal Eye Hospital for hydroxyapatite implantation were invited to take part in the study. Between July 1996 and June 1997, 55 patients agreed to participate. Procedures for general anaesthesia were standardised for each patient. The surgery was performed by the consultant (B.L.) and a number of surgeons in training. Standardised operative and postoperative protocols were followed.

An 11-point numerical ranking scale was used to measure patients' subjective experiences of pain. Patients were asked to score the degree of pain out of 10, 0 being 'no pain' and 10 being the 'worst pain imaginable'. Jensen et al. ${ }^{6}$ found that the scale was reliable and valid for measuring the intensity of surgical pain over a period of time. Patients with visual impairment appear able to understand and use the scale. ${ }^{7}$ In comparison, the visual analogue scale for assessing pain intensity is thought to be unsuitable for visually impaired patients because it requires functional vision to mark on or point to a line. Pain was measured once preoperatively, twice in the immediate postoperative period in the recovery room; then 2 
Table 1. Frequency of implant by gender

\begin{tabular}{lcrl}
\hline Implant & Male & Female & \multicolumn{1}{c}{ Total } \\
\hline Primary & $13(24 \%)$ & $7(13 \%)$ & $20(37 \%)$ \\
Secondary & $20(36 \%)$ & $15(27 \%)$ & $35(63 \%)$ \\
Total & $33(60 \%)$ & $22(40 \%)$ & $55(100 \%)$ \\
\hline
\end{tabular}

hourly for the rest of the day of surgery; and four times a day for 7 days until the first post-operative visit at 1 week post-operatively. One week was considered a sufficient length of time to conduct a detailed study on pain following surgery, particularly when patients' cooperation was sought for the completion of data collection forms.

Pain scores were entered onto a data collection form which also required information on the type, dose and time of analgesia administered and the location of pain. Other data were also collected that were thought to have an effect on pain: date of birth, gender, nausea, vomiting and infection. Nausea was measured on a 4-point ordinal scale: nil, mild, moderate and severe. Vomiting was recorded as either present or absent. The data collection sheet was devised for the study and piloted on five patients. Nurses were trained to complete the data information sheets reliably. It was found that with appropriate advice patients could be relied upon to complete these forms at home after discharge. Patients were also asked to elaborate on their experiences of pain and to comment on pre- and post-operative information.

Data were analysed using the Statistical Package for Social Sciences. Pain was not normally distributed. Pain was compared between implant groups using the MannWhitney $U$-test and within groups using the Wilcoxon test. The relationship between pre-operative pain and mean daily pain scores was explored using Spearman's Rank Correlation Coefficient. Chi-squared test (or Fisher's Exact test) was used to compare operation type and gender.

\section{Surgical technique}

The surgical technique was the same as that described by Ashworth et al. ${ }^{3}$ Briefly, the size of the hydroxyapatite implant to be used is measured with an acrylic sphere. Following enucleation, the implant is wrapped in donor sclera and inserted into a muscle cone created by the opening of the posterior Tenon's fascia. In primary implantation, the recti muscles and inferior oblique muscle are sutured to the donor sclera, whereas in secondary implantation the muscles were not identified; rather, the tissue in which they were thought to be contained was attached to the implant. The risk of post-operative haematoma and oedema is believed to be reduced when muscle dissection is avoided in secondary implantation. ${ }^{3}$

\section{Definition}

For the purposes of this study, primary implants were classed as those operations that included an enucleation. Secondary implants were categorised as those operations
Table 2. Range of pain scores by phase and implant

\begin{tabular}{lcc}
\hline Time & Primary implant & Secondary implant \\
\hline Pre-operation & $0-8$ & $0-4$ \\
Recovery & $0-9$ & $0-10$ \\
Day of surgery & $0-10$ & $0-10$ \\
Day 1 after surgery & $0-9$ & $0-8$ \\
Day 2 after surgery & $0-8$ & $0-10$ \\
Day 3 after surgery & $0-7$ & $0-10$ \\
Day 4 after surgery & $0-8$ & $0-8$ \\
Day 5 after surgery & $0-6$ & $0-7$ \\
Day 6 after surgery & $0-5$ & $0-7$ \\
Day 7 after surgery & $0-3$ & $0-4$ \\
\hline
\end{tabular}

NB: Day of surgery includes recovery readings.

in which the patient was anophthalmic or in which the patient already had an implant in situ that needed to be exchanged for a hydroxyapatite implant.

\section{Post-operative drug regime}

Post-operatively patients were prescribed: morphine 10-15 mg intramuscularly 4 hourly p.r.n., prochlorperazine $12.5 \mathrm{mg} 8$ hourly p.r.n., co-codamol (paracetamol $500 \mathrm{mg}$ and codeine $30 \mathrm{mg}$ ) orally 4 hourly p.r.n. (maximum 8 in 24 h), indomethacin $25 \mathrm{mg}$ orally t.d.s.; cephradine $500 \mathrm{mg}$ q.d.s. and g. chloramphenicol q.d.s.

\section{Results}

Thirty-three men and 22 women were studied. Table 1 contains the gender distribution by type of implant. The age range of patients was 20-72 years with a mean age of 39 years.

\section{Pre-operative pain}

As would be expected, pre-operative pain was significantly greater in patients who had primary hydroxyapatite implantation than in those who had secondary implantation $(p<0.001)$. Pre-operative pain in primary implant patients was positively correlated with post-operative daily mean pain scores on days 6 and 7 ( $p=0.005, r=0.62 ; p=0.004, r=0.647$, respectively).

\section{Post-operative pain}

Table 2 shows the range of pain scores by implant. Fig. 1 shows the pattern of mean pain scores on days 1 and 2 after surgery. By day 7,17 of $48(35.4 \%)$ patients were still recording pain (range $0-4)$. Fifteen $(28.8 \%$ ) patients

Table 3. Frequency of pain according to intensity and duration

\begin{tabular}{lccc}
\hline Pain & Long-lived & Short-lived & Total \\
\hline High & $15(28.8 \%)$ & $4(7.7 \%)$ & $19(36.5 \%)$ \\
Low & $18(34.6 \%)$ & $15(28.8 \%)$ & $33(63.5 \%)$ \\
Total & $33(63.5 \%)$ & $19(36.5 \%)$ & $52(100 \%)$ \\
\hline
\end{tabular}

High, scores 5-10 for a third of the readings of the first 3 days; Low, scores 0-4 for most of the readings, rarely reaching 7; Short-lived, pain score 0 for most of the last 4 days; Long-lived, pain score $>1$ for some of the readings of the last 4 days. 


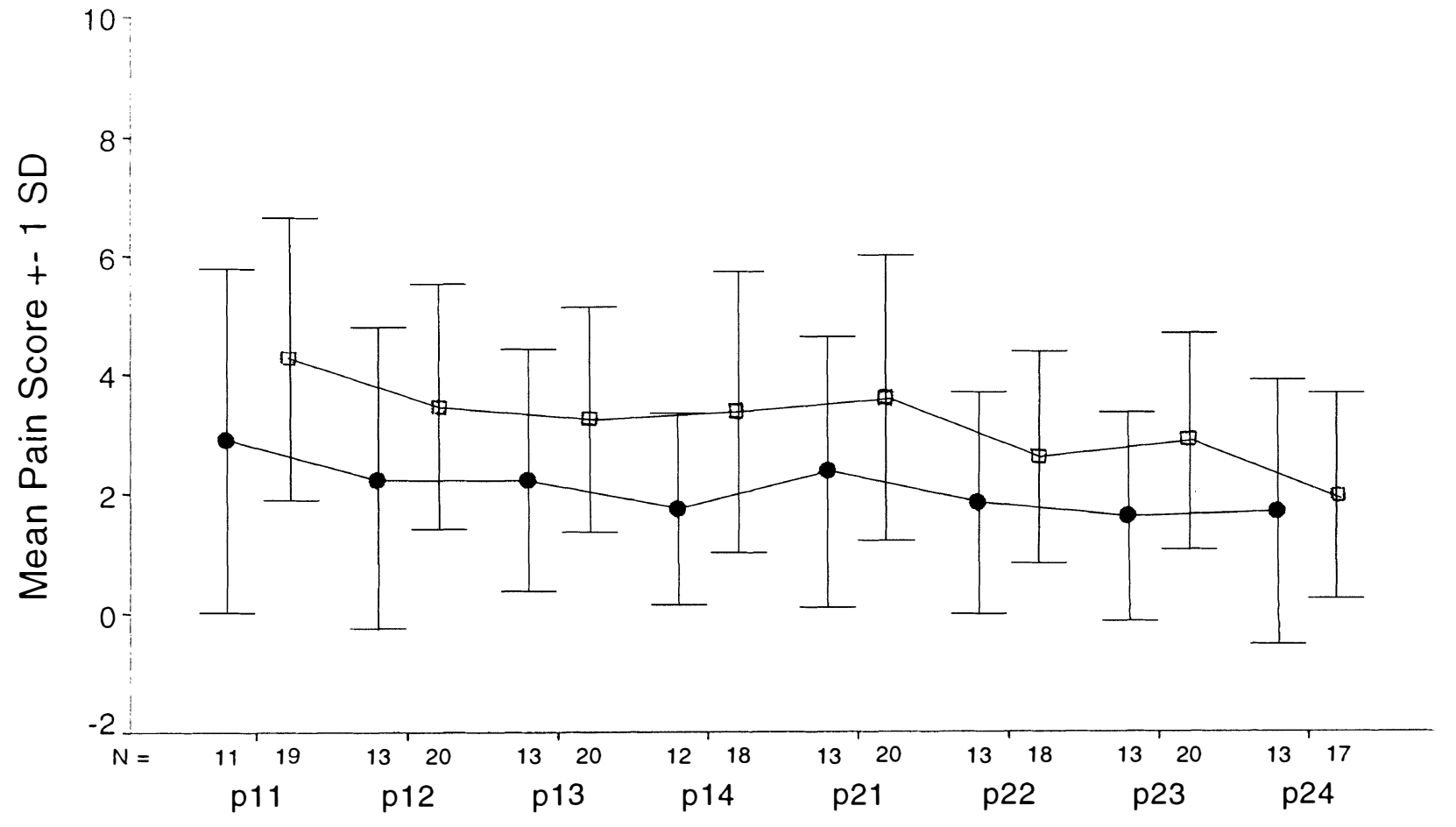

Time

Fig. $\mathbf{1}_{\mathbf{\varepsilon}}$ Pattern of mean pain scores over days 1 and 2 after surgery $(\mathrm{n}=35)$. Filled circles, primary implants; open squares, secondary implants.

experienced levels of pain in the range 5-10 for at least a third of the readings for the first 3 days and had pain that persisted for at least 7 days (Table 3).

\section{Gender and age}

There was no statistically significant difference in mean daily pain scores between men and women. Mean daily pain scores were the average of each patient's daily score. No statistically significant correlation was found between age and mean daily pain scores.

\section{Length of operation}

Length of surgery was found to be weakly associated with mean daily pain scores only on the day of surgery (Spearman's $r=0.291, p=0.036$ ). The length of surgery, excluding anaesthetic and recovery times, for primary implant patients was longer than for secondary implant patients (mean length of surgery $105.05 \mathrm{~min}$ vs $75.46 \mathrm{~min}$ respectively, $p=0.003$ ).

\section{Implant}

There was no significant difference between mean daily pain scores of primary and secondary implant patients. The third reading on the day of surgery was the only time when there was a significant difference in pain between primary and secondary patients (mean rank 30.66 vs 21.42 respectively, $p=0.0281$ ).

\section{Analgesia}

A few patients appear to have taken more than the recommended daily dose of analgesia. Patient 5 , for instance, self-medicated with flurbiprofen as well as indomethacin. She took both, three times a day for 6 days. Patient 19 reported that he took three co-proxamol every $2 \mathrm{~h}$ for $48 \mathrm{~h}$ in addition to the prescribed take-home medication!

\section{Post-operative nausea and vomiting}

There was no significant difference in pain scores between those who vomited and those who did not, up to and including day 3 . However, there were statistically

Table 4. Correlation between post-operative nausea and pain

\begin{tabular}{lcc}
\hline Time & Spearman's rho correlation coefficient & Significance (two-tailed) \\
\hline Day of surgery, third reading & 0.304 & $0.034^{*}$ \\
Day of surgery, fourth reading & 0.336 & $0.028^{*}$ \\
Day after surgery, first reading & 0.409 & $0.004^{* *}$ \\
Day after surgery, second reading & 0.014 & $0.014^{*}$ \\
Day after surgery, third reading & 0.470 & $0.000^{* *}$ \\
\hline
\end{tabular}

${ }^{*}$ Correlation significant at the 0.05 level; ${ }^{* *}$ correlation significant at the 0.01 level. 
significant correlations between nausea and pain twice on the day of surgery and three times on the day after surgery (Table 4 ).

\section{Post-operative infection}

There were no instances of post-operative infection.

\section{Discussion}

Pain is considered an important outcome indicator for day case and short-stay surgery worldwide. With increasing numbers of surgical cases being performed as day case or short stay, it is imperative that patients' pain is adequately managed in hospital and at home. ${ }^{8}$

Previous research demonstrates that patients who have undergone extraocular surgery are likely to experience more pain than those who have undergone intraocular surgery. ${ }^{7,9,10}$ Different methods of data collection make it difficult to carry out direct comparisons between the results of other studies. It appears, however, that on examination of pain scores, hydroxyapatite implant patients may have pain on a par with general surgical patients ${ }^{11,12}$ and with other extraocular patients ${ }^{9,10}$ over the first $24 \mathrm{~h}$. Pain scores on the day of and day after surgery appear to be greater than those reported for cataract surgery. ${ }^{10}$ Analysis of the data suggests that patients' levels of pain fell quite quickly over the first 3-4 days, which is dissimilar to typical pain scores of general surgical patients. ${ }^{13}$ Higher pre-operative pain was associated with persistent postoperative pain. It is proposed that pre-operative pain may have a 'carry-over' effect into the post-operative period caused by inflamed orbital tissues.

More surgical trauma is likely to occur in long operations and might explain why length of surgery was found to be weakly correlated with pain. In our study, primary implant patients were found to have longer operations than those who had secondary implants, whereas Cowper ${ }^{1}$ argues that the length of surgery in secondary implant patients is inevitably longer. The shorter operative times arose because secondary implant surgery did not involve the individual dissection and attachment of extraocular muscles. ${ }^{14}$ Primary implant patients also had significantly more pain postoperatively on one occasion. It appears, therefore, that primary implantation is more likely to be painful than secondary implantation with this particular surgical technique.

There are no reasons to suggest that pain is likely to be linked to the type of implant although, as yet, no direct comparisons have been made.

The data seem to corroborate the view that nausea is associated with pain rather than vomiting. ${ }^{15-17}$ Patients' comments also suggested that they attributed nausea either to high pain levels or to side effects of general anaesthesia and post-operative drug regimes. It would seem that, by controlling pain, nausea might also be managed.
The patients were occasionally asked to vacate their bed early on the first post-operative morning to provide a bed for pre-operative patients. The pattern of patients' pain demonstrates that pain is often worse on waking, particularly on the first day after surgery, and thus this is not the best time to discharge patients. The discharge arrangements of short-stay patients need to be carefully considered and should coincide with the daily optimum time of least pain and nausea. The fact that a few patients were taking more than the recommended dose of analgesia at home suggests that patients need to be sent home on adequate analgesia and with clear advice on how to manage their pain.

Methods for reducing post-operative pain might include soaking the implant in bupivacaine hydrochloride and/or instilling amethocaine hydrochloride drops. These local anaesthetics have a short duration of action and, since opiates are given anyway, are unlikely to affect pain scores. To instil regular anaesthetic eye drops post-operatively would mean disturbing the pressure bandage, which in turn may lead to more swelling - which in itself is thought to contribute to pain. Retrobulbar injections have also been suggested as another method of controlling postoperative pain; however, there is a small risk of haemorrhage that can seriously affect the outcome of surgery. At present the lack of evidence negates the suggestion of an optimal peri/post-operative pain management regime except to say that it should be tailored to the individual patient.

Future research might investigate the differences in pain between those patients who have an implant following evisceration and those who have an enucleation. Further work could usefully be undertaken to study the effect of pain and pain control on patients' ability to cope with the loss of an eye.

Although the sample size and survey design in this study prevent definitive conclusions it does indicate that this group of ophthalmic patients seems to suffer pain on the day of and after surgery on a par with that of general surgical patients. Some primary implant patients appear to experience more pain than those who have secondary implants, and this could be related to pre-operative pain. The financial pressure for reducing the number of nights patients spend in hospital should not outweigh the clinical needs of post-operative patients. Patients need to be given adequate analgesia to take home to prevent potentially dangerous self-prescription of drugs.

This study was supported with commitment and enthusiasm by patients, surgeons, anaesthetists and nurses. Their efforts are very greatly appreciated.

\section{References}

1. Cowper TR. Hydroxyapatite motility implants in ocular prosthetics. J Prosthetic Dentistry 1995;73:267-73.

2. Dutton JJ. Coralline hydroxyapatite as an ocular implant. Ophthalmology 1991;98:370-7. 
3. Ashworth J, Rhatigan M, Sampath R, Brammer R, Sunderland S, Leatherbarrow B. The hydroxyapatite orbital implant: a prospective study. Eye 1996;10:29-37.

4. Oestricher JH, Lui E, Berkowitz M. Complications of hydroxyapatite orbital implants. Ophthalmology 1997;104:324-9.

5. Shields CL, Shields JA, De Potter P, Singh AD. Problems with the hydroxyapatite orbital implant: experience with 250 consecutive cases. Br J Ophthalmol 1994;78:702-6.

6. Jensen MP, Karoly P, O'Riordan EF, Bland F, Burns RS. The subjective experience of acute pain. Clin J Pain 1989;5:153-9.

7. Waterman H, Grabham J, Obanye G. The incidence of pain in post-operative ophthalmic patients. Ophthalmic Nursing 1997;2:4-10.

8. Osborne GA, Rudkin GE. Outcome after day-case surgery in a major teaching hospital. Anaesth Intens Care 1993;21:822-7.

9. Van den Berg AA, Lambourne A, Yazji NS, Laghari NH. Vomiting after ophthalmic surgery. Anaesthesia 1987;42:270-6.
10. Van den Berg AA, Lambourne A, Clyburn PA. The oculoemetic reflex. Anaesthesia 1989;44:110-7.

11. Oates JD, Snowdon SL, Jayson DW. Failure of pain relief after surgery. Anaesthesia 1994;49:755-8.

12. Tittle M, McMillan S. Pain and pain-related side-effects in an ICU and on a surgical unit: nurses' management. Am J Crit Care 1994;3:25-30.

13. Seers K. Patients' perceptions of acute pain. In: WilsonBarnett J, Robinson S, editors. Directions in nursing research. London: Scutari, 19:Chap 12.

14. Massry GG, Holds JB. Complications of hydroxyapatite orbital implant. Ophthalmology 1997;104:1368-9.

15. Andrews PL. Physiology of nausea and vomiting. Br J Anaesth 1992;69(Suppl 1):s2-19.

16. Kenny GN. Risk factors for post-operative nausea and vomiting. Anaesthesia 1994;49(Suppl):6-10.

17. Watcha MF, White PF. Post-operative nausea and vomiting. Anesthesiology 1992;77:162-84. 